\title{
Pre and Post Emergence Application of Imazthapyr on N Uptake, Nodulation and Microbial Population of Chickpea Sown after Rice in Vertisols of C. G.
}

\author{
Anjum Ahmad*, Tapas Chowdhury, Sudhir Kumar Taunk, \\ Devendra Dewangan, and A.P. Singh
}

\author{
Department of Agronomy, Microbiology, Indira Gandhi Krishi Vishwa Vidyalaya, \\ Raipur - 492 012, India \\ *Corresponding author
}

\section{A B S T R A C T}

Keywords

Imazethapyr,

Nodulation,

Chickpea,

N Uptake,

Microbial activities.

Article Info

Accepted:

26 May 2017

Available Online:

10 June 2017
A field experiment was conducted at the Agronomy Research Farm of IGKV, Raipur, during the Rabi season of 2010-11 and 2011-12. Incorporation of tillage and weed management practices considerably improved the yield of chickpea interms of $\mathrm{N}$-uptake, number of nodules per plant and microbial activities of rice rhizosphere. Results indicated that, among the tillage management practices, higher $\mathrm{N}$ uptake, number of nodules and microbial activities were obtained with CT which was followed by MT and ZT. Among the various weed management practices, $\mathrm{N}$-uptake and number of nodules were maximum under one $\mathrm{HW}$ at 20 DAS, followed by the treatments of POE application of imazethapyr @ $90 \mathrm{~g} \mathrm{ha}-1$ and POE imazethapyr @ $80 \mathrm{~g}$ ha-1, respectively. Whereas, microbial population, basal soil respiration and dehydrogenase enzyme activity of experimental field was significantly higher under weedy check plot, followed by one HW at 20 DAS and POE application of imazethapyr @ $90 \mathrm{~g}$ ha-1 during both the years.

\section{Introduction}

Chickpea (Cicer arietinum L.) is an ancient crop and is grown in tropical, subtropical, and temperate regions of the country and in recent years. Chhattisgarh region is dominated by rice crop in rainy (kharif) season followed by cultivation of wheat, oilseed and pulses in winter (rabi) season. In Chhattisgarh, chickpea is cultivated in an area of about 3.20 Lakh ha with an average production of 2.12 Lakh tonnes and productivity of $663 \mathrm{~kg}$ ha-1. The average productivity of chickpea is still below one ton per hectare, which is considered low by any standards. As yield is a very complex character depending on number of component characters, the knowledge of the association between the yield and its components and among the components themselves is of immense practical value in making selections. In spite of the importance of this crop in our daily diet and in agricultural production system, the productivity of this crop is very low in India as well as in Chhattisgarh.

Weed competition is considered as one of the most important causes of low productivity and inferior quality of chickpea in Chhattisgarh. Considerable yield losses in 
chickpea recorded to the extent of 88 per cent if weeds are not controlled within critical growth period (Bhalla et al., 1998).

Appropriately selected herbicides may perform an important role in weed infestation reduction, increasing weeds resistance to herbicides, high cost and especially, negative effects of herbicides on environment have increased the need of non-chemical weed control in agro ecosystems (Augustin, 2003). Crop rotation, the tillage systems, application of agrochemicals and other agricultural practices affect the soil seed bank and weed flora (Marshall et al., 2003).

In an integrated approach, the development of cropping systems such as appropriate spatial arrangement and efficient tillage will help crops themselves to compete with weeds. Manipulation of cropping systems for the purpose of improving integrated weed management requires a good understanding of weed dynamics and influences of crop and soil-related factors on weed life cycles (Davis and Liebman, 2003). Weed flora have changed over the past century, with either increasing or decreasing species abundance depending on the management (Bagment, 2000).

Chickpea grown in succession with rice and soybean under irrigated condition infested heavily with weeds affected the growth and yield of chickpea. Crop losses of $90 \%$ are possible in weedy situations (Knights, 1991) and lack of registered post-emergence herbicides for broadleaf weeds reduces the options for weed management. Hand and mechanical weed control methods traditionally followed in the spring crop are not effective in winter sown chickpea besides being costly and uneconomical. Because of the sensitivity of chickpea to herbicides, most effective herbicides are pre-sowing and preemergence soil-acting chemicals and their efficacy is highly dependent on soil type, moisture, temperature and weed flora. Postemergence herbicides, particularly those for broad-leaf weeds are few. There is a need to identify more effective herbicides with broader spectrum of weed control and wide adaptability. An integrated approach involving herbicides and cultural practices to improve crop competitiveness is needed to develop effective and economic control measure.

\section{Materials and Methods}

A field experiment was conducted during rabi seasons of 2010-11 and 2011-12 at the Research cum Instructional Farm of Indira Gandhi Krishi Vishwavidyalaya, Raipur (Chhattisgarh). The soil of experimental field was clayey in texture, low in nitrogen, medium in phosphorus and high in potassium contents with neutral $\mathrm{pH}$. The experiment was laid out in Split Plot Design with three replications(Gomez and Gomez 1984). The treatments were divided into main and sub plots (tillage and weed management practices). Three tillage practices viz. conventional tillage (T1), minimum tillage (T2) and zero tillage (T3) in main plot Most mechanical weed control methods, such as hoeing, tillage, harrowing, torsion weeding, finger weeding and brush weeding, are used at very early weed growth stages (Singh, 2014; Kewat, 2014). and nine weed management practices as pendimethalin @ $1000 \mathrm{~g} \mathrm{ha}^{-1} \mathrm{PE}$ (W1), imazethapyr @ $80 \mathrm{~g} \mathrm{ha}^{-1}$ PE (W2), imazethapyr @ $90 \mathrm{~g} \mathrm{ha}^{-1} \mathrm{PE}$ (W3), imazethapyr@100 $\mathrm{g} \mathrm{ha}^{-1} \mathrm{PE}$ (W4) at 2 DAS, imazethapyr @70 g ha ${ }^{-1}$ POE (W5), imazethapyr @ 80 g ha ${ }^{-1}$ POE (W6), imazethapyr @90 $\mathrm{g} \mathrm{ha}^{-1}$ POE (W7) at 20 DAS, one hand weeding at 20 DAS (W8) and weedy check (W9), in sub plots. Removing weeds or patch of weeds by hand is often the most effective way to prevent that weed from spreading and therefore from becoming a 
serious problem (Zimdhal, 2007).The N, P, K through diamonium phosphate and muriate of potash were applied as basal at sowing of the crop. Fertilizers alter the nutrient level in the agro-ecosystems and therefore they may directly affect weed population dynamics and crop weed competitions (Robert et al., 2004; Babu and Jain, 2012).

Strong effects can be observed by manipulating fertilizer timing, dosage, and placement in order to reduce weed interference in crops (Dubey, 2014). Placement of fertilizer significantly reduced the density and dry biomass of weed and produced higher grain yield than broadcast method of fertilizer application (Pandey et al., 2006; Lodha et al., 2010). One protective irrigation gave at the time of sowing.

Optimum time and number of irrigation reduces the density and weight of weeds (Das and Yaduraju, 2007; Verma, 2014). The chickpea variety JG-226 was sown as test crop in 2nd fortnight of November 2010 and 2011 and harvesting was done in 1st fortnight of March 2011 and 2012, respectively.

\section{Nitrogen uptake by crop and weeds}

The sample of crop and weeds grain and stover were dried in oven at $60^{\circ} \mathrm{C}$ till constant weight after sun drying. $\mathrm{N}$ content (\%) was determined by Micro Kjeldahl method (Jackson, 1967). The nitrogen uptake was calculated for each treatment separately using the following formula.

Nitrogen uptake by grain $=\mathrm{N}$ Concentration $(\%)$ in grain x Grain yield $\left(\mathrm{kg} \mathrm{ha}^{-1}\right) / 100$

Nitrogen uptake by stover $=\mathrm{N}$ Concentration (\%) in stover x Stover yield $\left(\mathrm{kg} \mathrm{ha}^{-1}\right) / 100$

The uptake of $\mathrm{N}$ was expressed in $\mathrm{kg} \mathrm{ha}^{-1}$

\section{Number of nodules plant ${ }^{-1}$}

The number of nodules per plant were recorded at 20, 40 and 60 DAS. The roots removed from the 3 plants for root studies, were used to count the number of nodules. The nodules of each root were counted and mean value was noted as number of nodules plant $^{-1}$.

\section{Microbial analysis}

\section{Population count study}

Bacterial and fungal population was counted by using serial dilution technique (Subba Rao, 1988). One gm of soil sample was suspended in $9 \mathrm{ml}$ of sterile water in a dilution tube (Tuladhar, 1983) and shaken for $15 \mathrm{~min}$. This constituted $10^{-1}$ concentration. Using a fresh sterile pipette took $1 \mathrm{ml}$ of this suspension and $9 \mathrm{ml}$ sterile water was then added to get 10-2 dilution. The sequence was continued till a dilution of 10-7 was reached.

Different media was prepared for isolation of micro-organism. Thornton's Asparagine Mannitol agar media (Thornton, 1922) for bacteria and Rose Bengal agar media (Martin, 1950) for fungi were used, which were sterilized at $121^{\circ} \mathrm{C}$ for $15 \mathrm{~min} .1 \mathrm{ml}$ of desired solution of freshly mixed suspension was transferred into the sterile petridish using sterile tip of micro-pipette. $10-3$ to $10-5$ dilutions for fungi and 10-5 to 10-7 dilutions for bacteria were used. Subsequently, about $15 \mathrm{ml}$ of partially cooled appropriate medium was poured into each plate and carefully swirl to thoroughly mix the contents (Plate 3b). After the media got solidified invert the plates and kept in an incubator at respective incubation temperature for different microorganisms $\left(28^{\circ} \mathrm{C}\right.$ for fungi and $37^{\circ} \mathrm{C}$ for bacteria). After specified period of growth (48 hrs for bacteria and $96 \mathrm{hrs}$ for fungi), colonies were counted and population was enumerated 
by using formula given by Schmidt and Caldwell, 1967.

Number of bacteria / fungi in $1 \mathrm{gm}$ soil= No. of CFU x Dilution

Dry weight of $1 \mathrm{gm}$ moist soil $\mathrm{x}$ aliquot taken

\section{Basal Soil Respiration study}

This study was conducted to know the respiration rate of microflora present in the crop rhizosphere soil. Basal soil respiration was calculated by measuring the $\mathrm{CO}_{2}$ evolution rates (Anderson, 1982). $100 \mathrm{~g}$ soil (oven dry basis) was taken in 1L conical flask. Then water is added to bring its moisture content to field capacity. $20 \mathrm{ml}$ of $0.5 \mathrm{~N} \mathrm{NaOH}$ was taken in test tubes. The tubes were then hanged with the help of thread inside the conical flasks without touching the soil and kept the flasks air tight by rubber stoppers and note down the time. The flasks were kept in an incubator at $28^{\circ} \mathrm{C}$ for about 20 hrs. After incubation test tubes were taken out from the flask and noted down the time to calculate the period of incubation from the time as noted down above. Immediately transferred the $0.5 \mathrm{~N} \mathrm{NaOH}$ solution from the test tube to a $150 \mathrm{ml}$ conical flask. Several washings of the tubes were done for complete transfer $5 \mathrm{ml}$ of $3 \mathrm{~N} \mathrm{BaCl}_{2}$ solution and few drops of phenolphthalein indicator were added. Titrated the content with standard $0.5 \mathrm{~N} \mathrm{H}_{2} \mathrm{SO}_{4}$ slowly until the pink colour just disappears. After getting the end point recorded the exact amount of acid required for titration.

Soil respiration $(\mathrm{mg}$ of $\mathrm{CO} 2 / \mathrm{h} / 100 \mathrm{~g}$ soil $)=$ (B-V) NE/ hours of incubation

Where, $\mathrm{B}=$ Volume of acid $(\mathrm{ml})$ needed for the blank.

$\mathrm{V}=$ Volume of acid $(\mathrm{ml})$ needed for the $\mathrm{NaOH}$ exposed to soil.
$\mathrm{N}=$ Normality of acid.

$\mathrm{E}=$ Equivalent weight, i.e. 22.

\section{Dehydrogenase activity}

The procedure to evaluate the dehydrogenase activity of soil described by Klein et al., (1971). $1 \mathrm{gm}$ air dried soil sample was taken in a $15 \mathrm{ml}$ airtight screw capped test tube. 0.2 $\mathrm{ml}$ of 3\% TTC solution was added in each of the tubes to saturate the soil. $0.5 \mathrm{ml}$ of distilled water was also added in each tube. Gently tap the bottom of the tube to drive out all trapped oxygen so that a water seal was formed above the soil. No air bubbles were formed that was ensured. The tubes were incubated at $370 \mathrm{C}$ for $24 \mathrm{hrs}$. Then $10 \mathrm{ml}$ of methanol was added. Shake it vigorously and allowed to stand for $6 \mathrm{hrs}$. Clear pink coloured supernatant was withdrawn and readings were taken with a spectrophotometer. The amount of TPF formed was calculated from the standard curve drawn in the range of $10 \mathrm{mg}$ to $90 \mathrm{mg}$ $\mathrm{TPF} / \mathrm{ml}$.

\section{Results and Discussion}

\section{Nitrogen uptake by crop and weeds}

Data on quality parameters viz. nitrogen uptake table 2 indicated substantial variations due to tillage and weed management practices. The differences with respect to the nitrogen uptake were significant. Conventional tillage was found to contain maximum $\mathrm{N}$ uptake (by crop $29.66 \mathrm{~kg} \mathrm{ha}^{-1}$ nitrogen and by weed $33.72 \mathrm{~kg} \mathrm{ha}^{-1}$ nitrogen) and significantly higher than minimum and zero tillage during both the years. Hand weeding further excel all other treatments with respect to $\mathrm{N}$ uptake as it took maximum $\mathrm{N}$ uptake by crop (33.56 kg ha ${ }^{-1}$ nitrogen) and weeds (17.25 kg ha ${ }^{-1}$ nitrogen), which was significantly superior over pre-emergence and post-emergence herbicides (pendimethalin 
and imazethapyr) and weedy check plot. The weed-free treatment had more nitrogen uptake in grain and stalk than the weedy check during both the years. This was simply because of low shoot dry-matter production and low availability of these nutrients, as major amounts of nutrient were depleted by weeds. In weedy treatments, $37.5 \mathrm{~kg} \mathrm{~N}, 11.6$ $\mathrm{kg} \mathrm{P}$ and $38.3 \mathrm{~kg} \mathrm{~K} / \mathrm{ha}$ were depleted in comparison to full-season weed-free conditions. The results are in conformity with the findings of Vengris et al., (1953), who reported vigorous growth and higher biomass of weeds resulted in more nutrient depletion.

\section{Number of nodules plant ${ }^{-1}$}

The tillage and weed management practices significantly influenced the number of nodules per plant at all the growth stages of chickpea crop except 20 DAS during both the years. Tillage practices enhanced the number of nodules per plant significantly in chickpea during all the stages of crop growth. Conventional tillage proved to be favourable for producing significantly greater number of root nodules and it was followed by minimum and zero tillage, in descending order, in both the years. The treatment of one hand weeding at 20 DAS (W8) superseded all the herbicidal treatments with respect to maximum nodulation, as it contains 10.56, 26.33 and 38.75 root nodules per plant at 20, 40, 60 DAS, respectively. Among the herbicidal treatments, imazethapyr @ $90 \mathrm{~g} \mathrm{ha}^{-1}$ POE (W7) produced significantly greater number of nodules per plant as compared to other treatments at all the growth stages during both the years. The treatments of imazethapyr @ 80 and $70 \mathrm{~g} \mathrm{ha}^{-1}$ as post-emergent were next, in order, and produced statistically greater number of nodules as compared to weedy check. Other herbicidal treatments also proved better than weedy check at all the growth stages under study during both the years (Table 3). Hand-weeding twice recorded the maximum number of nodules/plant. Ahlawat et al., (1985). All the weed-control treatments proved superior to the unweeded check treatment. Hand weeding at 25 and 45 days was found most promising. Malik et al., (1988) also reported similar results. Results indicated that inter-culture + hand weeding done at 20 and 40 DAS recorded significantly higher nodulation as compared to rest of the treatments except pendimethalin@0.75 kg/ha. Similar results were also reported by Singh and Singh (1998), wherein, they found the highest nodulation in hand weeded plot and the lowest in fluchloralin treated plot. These results are in agreement with the results of Rajput et al., (1998) who observed higher nodule weight under hand weeded and pendimethalin application over unweeded control in chickpea.

\section{Micro-flora studies}

\section{Bacterial population study}

The result on total bacterial population on rhizosphere soil as affected by different tillage and weed management practices were recorded at definite interval and tabulated in table 1 .

In rice-chickpea cropping sequence, the bacterial population study as affected by different tillage management practices, which indicated that tillage system did not impart any effect on batcterial population after harvest of the crop. Similar findings were also reported by Singh et al., (2007) who clearly mentioned that relatively higher availability of soil organic matter at lower soil profile under conventional tillage may be due to even distribution of crop residues and other nutrients throughout the plough zone. This may possibly account for observed higher counts of soil microflora at lower $(7.5-15 \mathrm{~cm}$.) soil zone under conventional tillage than the 
minimum tillage. Brady (1985) reported that tillage facilitates the aeration of soil hence the microbial population increases. Singh et al., (2007) also found higher microbial populations under conventional tillage system at lower soil depth (7.5-15 cm.). Janusauskaite et al., (2013) demonstrated that bacteria and fungi decreased in no tillage system by 25.5 and $22.7 \%$, respectively in comparison to conventional tillage.

It can be concluded that conventional tillage system provides stimulating effects for microbial growth due to uniformly distributed residues in the arable layer and increases the rate of supplied oxygen to soil micro sites. The data related to total bacterial population in rice-chickpea cropping sequence revealed that among the different weed management practices, one hand weeding at 20 DAS was found effective to increase bacterial population superior over other treatments under study. These observations are in close agreement with Singh (1990) who reported that population of bacteria were affected with pre and post emergence application of herbicides and these adverse effects gradually reduced with passage of time. Sebiomo et al., (2010) who found that the herbicide treatments had significant effect on percent organic matter of the soils treated with herbicides, which reduced significantly as compared to control. The total bacterial population in rhizosphere soil of chickpea found significantly lower in herbicide treated plots compared to hand weeded and weedy check plots in all the growth period of crop.

Table.1 Effect of tillage practices and weed control measures on total bacterial population (X $10^{7} \mathrm{~g}^{-1}$ soil) of rhizosphere soil at different growth stages of chickpea after harvest of rice

\begin{tabular}{|c|c|c|c|c|c|c|}
\hline \multirow[t]{3}{*}{ Treatments } & \multicolumn{6}{|c|}{ Days after sowing } \\
\hline & \multicolumn{3}{|c|}{ At Initial } & \multicolumn{3}{|c|}{ At harvest } \\
\hline & $\begin{array}{l}2010- \\
2011\end{array}$ & $\begin{array}{l}2011- \\
2012\end{array}$ & Mean & $\begin{array}{l}2010- \\
2011\end{array}$ & $\begin{array}{l}2011- \\
2012\end{array}$ & Mean \\
\hline $\begin{array}{l}\text { Main Plot: Tillage management } \\
\mathrm{T}_{1} \text { : Conventional }\end{array}$ & 0.708 & 0.344 & 0.526 & 2.379 & 1.228 & 1.804 \\
\hline $\mathrm{T}_{2}:$ Minimum & 0.667 & 0.331 & 0.499 & 2.360 & 1.124 & 1.742 \\
\hline $\mathrm{T}_{3}:$ Zero & 0.582 & 0.328 & 0.455 & 2.355 & 1.015 & 1.685 \\
\hline $\begin{array}{l}\mathrm{SEm} \pm \\
\operatorname{CD}(\mathbf{P}=0.05)\end{array}$ & $\begin{array}{l}0.043 \\
\text { NS }\end{array}$ & $\begin{array}{l}0.005 \\
\text { NS }\end{array}$ & & $\begin{array}{l}0.030 \\
\text { NS }\end{array}$ & $\begin{array}{c}0.060 \\
\text { NS }\end{array}$ & \\
\hline $\begin{array}{l}\text { Sub Plot: Weed management } \\
\mathrm{W}_{1} \text { : Pendimethalin @ } 1000 \mathrm{~g} / \mathrm{ha} \mathrm{PE}\end{array}$ & 0.650 & 0.403 & 0.527 & 2.278 & 1.160 & 1.719 \\
\hline $\mathrm{W}_{2}:$ Imazethapyr @80 g/ha PE & 0.654 & 0.463 & 0.559 & 2.331 & 1.183 & 1.757 \\
\hline W ${ }_{3}$ Imazethapyr @ 90 g/ha PE & 0.656 & 0.304 & 0.480 & 2.234 & 1.177 & 1.706 \\
\hline W & 0.650 & 0.250 & 0.450 & 2.178 & 1.161 & 1.670 \\
\hline $\mathrm{W}_{5}$ : Imazethapyr @ $70 \mathrm{~g} / \mathrm{ha} \mathrm{POE}$ & 0.657 & 0.312 & 0.485 & 2.424 & 1.150 & 1.787 \\
\hline $\mathrm{W}_{6}$ : Imazethapyr @ $80 \mathrm{~g} / \mathrm{ha} \mathrm{POE}$ & 0.659 & 0.176 & 0.418 & 2.314 & 0.998 & 1.656 \\
\hline $\mathrm{W}_{7:}$ Imazethapyr @ 90 g/ha POE & 0.647 & 0.143 & 0.395 & 2.144 & 0.888 & 1.516 \\
\hline $\mathrm{W}_{8:}$ One hand weeding at $20 \mathrm{DAS}$ & 0.650 & 0.476 & 0.563 & 2.638 & 1.163 & 1.901 \\
\hline $\mathrm{W}_{9}$ : Weedy Check & 0.652 & 0.483 & 0.568 & 2.644 & 1.220 & 1.932 \\
\hline $\begin{array}{l}\mathrm{S} \mathrm{Em} \pm \\
\mathrm{CD}(\mathrm{P}=0.05)\end{array}$ & $\begin{array}{l}0.074 \\
\text { NS }\end{array}$ & $\begin{array}{l}0.098 \\
\text { NS }\end{array}$ & & $\begin{array}{l}0.175 \\
\text { NS }\end{array}$ & $\begin{array}{l}0.175 \\
\text { NS }\end{array}$ & \\
\hline
\end{tabular}


Table.2 Nitrogen uptake by chickpea after harvest of rice as influenced by different tillage and weed management practices

\begin{tabular}{|c|c|c|c|c|c|c|}
\hline \multirow[t]{3}{*}{ Treatments } & \multicolumn{6}{|c|}{ N Uptake (Kg ha $\left.{ }^{-1}\right)$} \\
\hline & \multicolumn{3}{|c|}{ Crop } & \multicolumn{3}{|c|}{ weed } \\
\hline & 2010-2011 & 2011-2012 & Mean & 2010-2011 & 2011-2012 & Mean \\
\hline $\begin{array}{l}\text { Main Plot: Tillage management } \\
\mathrm{T}_{1} \text { : Conventional }\end{array}$ & 31.17 & 28.14 & 29.66 & 34.98 & 32.45 & 33.72 \\
\hline $\mathrm{T}_{2}:$ Minimum & 28.61 & 25.59 & 27.10 & 33.02 & 29.88 & 31.45 \\
\hline $\mathrm{T}_{3}:$ Zero & 25.33 & 22.66 & 23.99 & 30.90 & 27.53 & 29.21 \\
\hline S Em \pm & 0.94 & 0.94 & & 1.02 & 1.22 & \\
\hline $\mathrm{CD}(\mathbf{P}=\mathbf{0 . 0 5})$ & 3.71 & 3.71 & & 4.01 & 4.78 & \\
\hline Sub Plot: Weed management & & & & & & \\
\hline W W $_{1}$ Pendimethalin@1000 g/ha PE & 26.83 & 24.48 & 25.66 & 31.09 & 29.46 & 30.28 \\
\hline $\mathrm{W}_{2}$ : Imazethapyr @ $80 \mathrm{~g} / \mathrm{ha} \mathrm{PE}$ & 24.62 & 22.15 & 29.39 & 30.25 & 27.66 & 28.96 \\
\hline $\mathrm{W}_{3}$ : Imazethapyr @90 g/ha PE & 26.47 & 23.79 & 25.13 & 32.37 & 29.67 & 31.02 \\
\hline 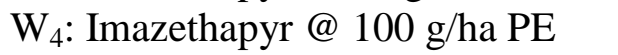 & 29.58 & 26.47 & 28.03 & 34.40 & 31.35 & 32.88 \\
\hline $\mathrm{W}_{5}$ : Imazethapyr @ $70 \mathrm{~g} / \mathrm{ha}$ POE & 30.48 & 27.36 & 28.92 & 35.74 & 32.46 & 34.10 \\
\hline W6: Imazethapyr @ 80 g/ha POE & 32.01 & 28.72 & 30.37 & 34.30 & 30.65 & 32.48 \\
\hline $\mathrm{W}_{7:}$ Imazethapyr @ $90 \mathrm{~g} / \mathrm{ha}$ POE & 33.22 & 29.72 & 31.47 & 33.65 & 28.74 & 31.19 \\
\hline $\mathrm{W}_{8:}$ One hand weeding at $20 \mathrm{DAS}$ & 35.52 & 31.59 & 33.56 & 18.12 & 16.38 & 17.25 \\
\hline $\mathrm{W}_{9}$ : Weedy Check & 16.58 & 14.90 & 15.74 & 46.77 & 43.20 & 44.99 \\
\hline $\mathbf{S ~ E m} \pm$ & 1.54 & 1.54 & & 1.42 & 1.46 & \\
\hline $\mathrm{CD}(\overline{\mathrm{P}}=\mathbf{0 . 0 5})$ & 4.37 & 4.37 & & 4.03 & 4.16 & \\
\hline
\end{tabular}


Table.3 Number of nodules plant ${ }^{-1}$ of chickpea after the harvest of rice at different growth stages as influenced by Different tillage and weed management practices

\begin{tabular}{|c|c|c|c|c|c|c|c|c|c|}
\hline \multirow[t]{2}{*}{ Treatments } & \multicolumn{3}{|c|}{20 DAS } & \multicolumn{3}{|c|}{40 DAS } & \multicolumn{3}{|c|}{60 DAS } \\
\hline & $\begin{array}{l}2010- \\
2011\end{array}$ & $\begin{array}{l}2011- \\
2012\end{array}$ & Mean & $\begin{array}{r}2010- \\
2011\end{array}$ & $\begin{array}{l}2011- \\
2012\end{array}$ & Mean & $\begin{array}{l}2010- \\
2011\end{array}$ & $\begin{array}{l}2011- \\
2012\end{array}$ & Mean \\
\hline $\begin{array}{l}\text { Main Plot: Tillage management } \\
\mathrm{T}_{1} \text { : Conventional } \\
\mathrm{T}_{2}: \text { Minimum } \\
\mathrm{T}_{3}: \text { Zero }\end{array}$ & $\begin{array}{l}11.06 \\
10.44 \\
10.23\end{array}$ & $\begin{array}{l}9.08 \\
8.53 \\
8.37\end{array}$ & $\begin{array}{c}10.07 \\
9.49 \\
9.30 \\
\end{array}$ & $\begin{array}{l}23.41 \\
21.82 \\
20.82\end{array}$ & $\begin{array}{l}20.26 \\
18.28 \\
16.72 \\
\end{array}$ & $\begin{array}{l}21.84 \\
20.05 \\
18.77 \\
\end{array}$ & $\begin{array}{l}32.24 \\
30.43 \\
29.39 \\
\end{array}$ & $\begin{array}{l}30.09 \\
27.85 \\
25.80\end{array}$ & $\begin{array}{l}31.17 \\
29.14 \\
27.60\end{array}$ \\
\hline $\begin{array}{l}\mathrm{S} \mathrm{Em}+ \\
\mathrm{CD}(\mathrm{P}=0.05)\end{array}$ & $\begin{array}{l}0.37 \\
\mathbf{1 . 4 4} \\
\end{array}$ & $\begin{array}{l}0.35 \\
\mathbf{1 . 3 7} \\
\end{array}$ & & $\begin{array}{l}0.59 \\
\mathbf{2 . 3 5} \\
\end{array}$ & $\begin{array}{l}0.51 \\
\mathbf{1 . 9 9} \\
\end{array}$ & & $\begin{array}{l}0.64 \\
2.51 \\
\end{array}$ & $\begin{array}{l}0.97 \\
\mathbf{3 . 8 1}\end{array}$ & \\
\hline $\begin{array}{l}\text { Sub Plot: Weed management } \\
\mathrm{W}_{1} \text { : Pendimethalin @ } 1000 \mathrm{~g} / \mathrm{ha} \mathrm{PE} \\
\mathrm{W}_{2} \text { : Imazethapyr @ } 80 \mathrm{~g} / \mathrm{ha} \mathrm{PE} \\
\mathrm{W}_{3} \text { : Imazethapyr @ } 90 \mathrm{~g} / \mathrm{ha} \mathrm{PE} \\
\mathrm{W}_{4} \text { : Imazethapyr @ } 100 \mathrm{~g} / \mathrm{ha} \mathrm{PE} \\
\mathrm{W}_{5} \text { : Imazethapyr @ } 70 \mathrm{~g} / \mathrm{ha} \mathrm{POE} \\
\mathrm{W}_{6} \text { : Imazethapyr @ } 80 \mathrm{~g} / \mathrm{ha} \mathrm{POE} \\
\mathrm{W}_{7} \text { Imazethapyr @ } 90 \mathrm{~g} / \mathrm{ha} \text { POE } \\
\mathrm{W}_{8} \text { : One hand weeding at } 20 \text { DAS } \\
\mathrm{W}_{9} \text { : Weedy Check }\end{array}$ & $\begin{array}{l}10.04 \\
8.58 \\
9.64 \\
10.02 \\
10.44 \\
11.06 \\
11.50 \\
11.80 \\
12.12\end{array}$ & $\begin{array}{l}8.32 \\
7.86 \\
7.89 \\
8.30 \\
8.54 \\
9.12 \\
9.27 \\
9.31 \\
9.34\end{array}$ & $\begin{array}{c}9.18 \\
8.22 \\
8.77 \\
9.16 \\
9.49 \\
10.09 \\
10.39 \\
10.56 \\
10.73\end{array}$ & $\begin{array}{l}18.92 \\
18.68 \\
19.76 \\
21.26 \\
23.90 \\
24.98 \\
26.60 \\
29.48 \\
14.58\end{array}$ & $\begin{array}{l}16.36 \\
14.65 \\
17.89 \\
18.89 \\
20.26 \\
20.81 \\
21.59 \\
23.18 \\
12.51\end{array}$ & $\begin{array}{l}17.64 \\
16.67 \\
18.83 \\
20.08 \\
22.08 \\
22.90 \\
24.10 \\
26.33 \\
13.55\end{array}$ & $\begin{array}{l}27.64 \\
26.49 \\
28.09 \\
30.04 \\
32.53 \\
34.76 \\
35.82 \\
40.98 \\
19.82\end{array}$ & $\begin{array}{l}24.24 \\
22.64 \\
25.84 \\
28.56 \\
29.44 \\
32.61 \\
33.75 \\
36.52 \\
17.60\end{array}$ & $\begin{array}{l}25.94 \\
24.57 \\
26.97 \\
29.30 \\
30.99 \\
33.69 \\
34.79 \\
38.75 \\
18.71\end{array}$ \\
\hline $\begin{array}{l}\mathrm{S} \mathrm{Em} \pm \\
\mathrm{CD}(\mathbf{P}=0.05)\end{array}$ & $\begin{array}{l}0.58 \\
\mathbf{1 . 6 5}\end{array}$ & $\begin{array}{l}0.59 \\
\mathbf{1 . 6 8}\end{array}$ & & $\begin{array}{l}0.94 \\
\mathbf{2 . 6 7}\end{array}$ & $\begin{array}{l}0.66 \\
\mathbf{1 . 8 7}\end{array}$ & & $\begin{array}{l}1.00 \\
\mathbf{2 . 8 4}\end{array}$ & $\begin{array}{l}1.20 \\
\mathbf{3 . 4 1}\end{array}$ & \\
\hline
\end{tabular}


Table.4 Effect of tillage practices and weed control measures on Basal soil respiration $(\mathrm{mg} \mathrm{CO} / \mathrm{h} / 100 \mathrm{~g}$ soil) Of rhizosphere soil at different growth stages of chickpea after harvest of rice

\begin{tabular}{|c|c|c|c|c|c|c|c|c|c|c|c|c|}
\hline \multirow[t]{3}{*}{ Treatments } & \multicolumn{12}{|c|}{ Days after sowing } \\
\hline & \multicolumn{3}{|c|}{10} & \multicolumn{3}{|c|}{30} & \multicolumn{3}{|c|}{$\mathbf{5 0}$} & \multicolumn{3}{|c|}{ At harvest } \\
\hline & $\begin{array}{l}2010- \\
2011\end{array}$ & $\begin{array}{l}2011- \\
2012\end{array}$ & Mean & $\begin{array}{l}2010- \\
2011\end{array}$ & $\begin{array}{l}2011- \\
2012\end{array}$ & Mean & $\begin{array}{l}2010- \\
2011\end{array}$ & $\begin{array}{l}2011- \\
2012\end{array}$ & Mean & $\begin{array}{l}2010- \\
2011\end{array}$ & $\begin{array}{l}2011- \\
2012\end{array}$ & Mean \\
\hline $\begin{array}{l}\text { Main Plot: Tillage management } \\
\mathrm{T}_{1} \text { : Conventional }\end{array}$ & 0.243 & 0.238 & 0.241 & 0.339 & 0.294 & 0.317 & 0.310 & 0.300 & 0.305 & 0.212 & 0.206 & 0.209 \\
\hline $\mathrm{T}_{2}:$ Minimum & 0.226 & 0.221 & 0.224 & 0.319 & 0.288 & 0.304 & 0.296 & 0.286 & 0.291 & 0.210 & 0.204 & 0.207 \\
\hline $\mathrm{T}_{3}:$ Zero & 0.208 & 0.202 & 0.205 & 0.299 & 0.276 & 0.288 & 0.277 & 0.267 & 0.272 & 0.204 & 0.200 & 0.202 \\
\hline $\begin{array}{l}\mathrm{SEm} \pm \\
\mathrm{CD}(\mathrm{P}=0.05)\end{array}$ & $\begin{array}{c}0.002 \\
\mathbf{0 . 0 1}\end{array}$ & $\begin{array}{c}0.006 \\
\mathbf{0 . 0 2}\end{array}$ & & $\begin{array}{c}0.009 \\
\mathbf{0 . 0 3}\end{array}$ & $\begin{array}{c}0.009 \\
\mathbf{0 . 0 3}\end{array}$ & & $\begin{array}{c}0.009 \\
\text { NS }\end{array}$ & $\begin{array}{c}0.009 \\
\text { NS }\end{array}$ & & $\begin{array}{c}0.002 \\
\text { NS }\end{array}$ & $\begin{array}{c}0.002 \\
\text { NS }\end{array}$ & \\
\hline Sub Plot: Weed management & & & & & & & & & & & & \\
\hline $\mathrm{W}_{1}$ : Pendimethalin @ $1000 \mathrm{~g} / \mathrm{ha} \mathrm{PE}$ & 0.151 & 0.139 & 0.145 & 0.329 & 0.295 & 0.312 & 0.404 & 0.394 & 0.399 & 0.212 & 0.207 & 0.210 \\
\hline W2: Imazethapyr @ 80 g/ha PE & 0.165 & 0.151 & 0.158 & 0.335 & 0.300 & 0.318 & 0.411 & 0.399 & 0.405 & 0.215 & 0.210 & 0.213 \\
\hline W: Imazethapyr @ 90 g/ha PE & 0.149 & 0.144 & 0.147 & 0.328 & 0.293 & 0.311 & 0.399 & 0.389 & 0.394 & 0.212 & 0.206 & 0.209 \\
\hline W & 0.146 & 0.141 & 0.144 & 0.321 & 0.290 & 0.306 & 0.387 & 0.375 & 0.381 & 0.208 & 0.204 & 0.206 \\
\hline W5: Imazethapyr @ 70 g/ha POE & 0.299 & 0.288 & 0.294 & 0.296 & 0.263 & 0.280 & 0.195 & 0.194 & 0.195 & 0.198 & 0.193 & 0.196 \\
\hline $\mathrm{W}_{6}$ : Imazethapyr @ $80 \mathrm{~g} / \mathrm{ha} \mathrm{POE}$ & 0.293 & 0.281 & 0.287 & 0.288 & 0.256 & 0.272 & 0.194 & 0.185 & 0.190 & 0.195 & 0.190 & 0.193 \\
\hline $\mathrm{W}_{7:}$ Imazethapyr @ $90 \mathrm{~g} / \mathrm{ha} \mathrm{POE}$ & 0.233 & 0.271 & 0.252 & 0.281 & 0.250 & 0.266 & 0.196 & 0.184 & 0.190 & 0.193 & 0.188 & 0.191 \\
\hline $\mathrm{W}_{8:}$ One hand weeding at $20 \mathrm{DAS}$ & 0.294 & 0.285 & 0.290 & 0.344 & 0.310 & 0.327 & 0.217 & 0.205 & 0.211 & 0.220 & 0.213 & 0.217 \\
\hline $\mathrm{W}_{9}$ : Weedy Check & 0.298 & 0.286 & 0.292 & 0.349 & 0.318 & 0.334 & 0.244 & 0.233 & 0.239 & 0.225 & 0.218 & 0.222 \\
\hline $\begin{array}{l}\mathrm{SEm} \pm \\
\mathrm{CD}(\mathbf{P}=0.05)\end{array}$ & $\begin{array}{c}0.004 \\
\mathbf{0 . 0 1}\end{array}$ & $\begin{array}{c}0.009 \\
\mathbf{0 . 0 3}\end{array}$ & & $\begin{array}{c}0.013 \\
\mathbf{0 . 0 4}\end{array}$ & $\begin{array}{c}0.013 \\
\mathbf{0 . 0 4}\end{array}$ & & $\begin{array}{c}0.014 \\
\mathbf{0 . 0 4}\end{array}$ & $\begin{array}{c}0.014 \\
\mathbf{0 . 0 4}\end{array}$ & & $\begin{array}{c}0.004 \\
\text { NS }\end{array}$ & $\begin{array}{c}0.004 \\
\text { NS }\end{array}$ & \\
\hline
\end{tabular}


Table.5 Effect of tillage practices and weed control measures on Dehydrogenase activity ( $\mu$ g TPF/h/g soil)

Of rhizosphere soil at different growth stages of chickpea after harvest of rice

\begin{tabular}{|c|c|c|c|c|c|c|c|c|c|c|c|c|}
\hline \multirow[t]{3}{*}{ Treatments } & \multicolumn{12}{|c|}{ Days after sowing } \\
\hline & \multicolumn{3}{|c|}{10} & \multicolumn{3}{|c|}{30} & \multicolumn{3}{|c|}{$\mathbf{5 0}$} & \multicolumn{3}{|c|}{ At harvest } \\
\hline & $\begin{array}{l}2010- \\
2011\end{array}$ & $\begin{array}{l}2011- \\
2012\end{array}$ & Mean & $\begin{array}{l}2010- \\
2011\end{array}$ & $\begin{array}{l}2011- \\
2012\end{array}$ & Mean & $\begin{array}{l}2010- \\
2011\end{array}$ & $\begin{array}{l}2011- \\
2012\end{array}$ & Mean & $\begin{array}{l}2010- \\
2011\end{array}$ & $\begin{array}{l}2011- \\
2012\end{array}$ & Mean \\
\hline $\begin{array}{l}\text { Main Plot: Tillage management } \\
\mathrm{T}_{1} \text { : Conventional }\end{array}$ & 12.76 & 10.73 & 11.75 & 32.53 & 30.47 & 31.50 & 55.76 & 52.10 & 53.93 & 29.42 & 25.74 & 27.58 \\
\hline $\mathrm{T}_{2}:$ Minimum & 10.74 & 8.71 & 9.73 & 30.56 & 28.44 & 29.50 & 53.54 & 50.21 & 51.88 & 28.31 & 25.66 & 26.99 \\
\hline $\mathrm{T}_{3}:$ Zero & 8.77 & 6.72 & 7.75 & 28.56 & 26.46 & 27.51 & 52.87 & 49.10 & 50.99 & 26.25 & 24.52 & 25.39 \\
\hline $\begin{array}{l}\mathrm{SEm} \pm \\
\mathrm{CD}(\mathrm{P}=0.05)\end{array}$ & $\begin{array}{l}0.28 \\
\mathbf{1 . 0 9}\end{array}$ & $\begin{array}{l}0.22 \\
\mathbf{0 . 8 7}\end{array}$ & & $\begin{array}{l}1.08 \\
4.24\end{array}$ & $\begin{array}{l}1.01 \\
3.97\end{array}$ & & $\begin{array}{l}1.66 \\
\text { NS }\end{array}$ & $\begin{array}{l}0.84 \\
\text { NS }\end{array}$ & & $\begin{array}{l}0.81 \\
\text { NS }\end{array}$ & $\begin{array}{l}0.31 \\
\text { NS }\end{array}$ & \\
\hline Sub Plot: Weed management & & & & & & & & & & & & \\
\hline $\mathrm{W}_{1}$ : Pendimethalin @ $1000 \mathrm{~g} / \mathrm{ha} \mathrm{PE}$ & 9.34 & 7.17 & 8.26 & 26.45 & 24.21 & 25.33 & 72.15 & 68.93 & 70.54 & 29.06 & 29.02 & 29.04 \\
\hline W2: Imazethapyr @ 80 g/ha PE & 10.36 & 8.34 & 9.35 & 27.42 & 25.32 & 26.37 & 75.09 & 71.84 & 73.47 & 30.96 & 28.15 & 29.56 \\
\hline W: Imazethapyr @ 90 g/ha PE & 8.03 & 6.16 & 7.10 & 25.31 & 23.14 & 24.23 & 71.53 & 66.69 & 69.11 & 28.88 & 25.98 & 27.43 \\
\hline W & 8.00 & 6.09 & 7.05 & 23.47 & 21.27 & 22.37 & 68.17 & 60.83 & 64.50 & 27.89 & 25.02 & 26.46 \\
\hline W5: Imazethapyr @ $70 \mathrm{~g} / \mathrm{ha}$ POE & 12.20 & 10.12 & 11.16 & 12.14 & 10.10 & 11.12 & 11.14 & 9.21 & 10.18 & 25.08 & 22.44 & 23.76 \\
\hline $\mathrm{W}_{6}$ : Imazethapyr @ $80 \mathrm{~g} / \mathrm{ha}$ POE & 12.22 & 10.16 & 11.19 & 10.06 & 9.34 & 9.70 & 9.61 & 8.49 & 9.05 & 22.03 & 19.28 & 20.66 \\
\hline $\mathrm{W}_{7:}$ Imazethapyr @ $90 \mathrm{~g} / \mathrm{ha} \mathrm{POE}$ & 12.21 & 10.15 & 11.18 & 8.14 & 7.21 & 7.68 & 7.75 & 6.36 & 7.06 & 21.14 & 18.93 & 20.04 \\
\hline $\mathrm{W}_{8:}$ One hand weeding at $20 \mathrm{DAS}$ & 12.22 & 10.14 & 11.18 & 68.83 & 65.16 & 67.00 & 79.73 & 74.83 & 77.28 & 32.78 & 28.97 & 30.88 \\
\hline $\mathrm{W}_{9}$ : Weedy Check & 12.22 & 10.15 & 11.19 & 73.13 & 70.36 & 71.75 & 91.31 & 87.03 & 89.17 & 34.11 & 30.01 & 32.06 \\
\hline $\begin{array}{l}\mathrm{S} \mathrm{Em} \pm \\
\mathrm{CD}(\mathbf{P}=0.05)\end{array}$ & $\begin{array}{l}0.46 \\
\mathbf{1 . 3 2}\end{array}$ & $\begin{array}{l}0.38 \\
\mathbf{1 . 0 8}\end{array}$ & & $\begin{array}{l}1.62 \\
4.62\end{array}$ & $\begin{array}{l}1.53 \\
4.36\end{array}$ & & $\begin{array}{l}2.65 \\
\mathbf{7 . 5 3}\end{array}$ & $\begin{array}{l}1.38 \\
\mathbf{3 . 9 1}\end{array}$ & 2.02 & $\begin{array}{l}1.25 \\
\text { NS }\end{array}$ & $\begin{array}{l}0.54 \\
\text { NS }\end{array}$ & \\
\hline
\end{tabular}


Microbiological study (Basal soil respiration)

The observations on Basal Soil Respiration (BSR) as influenced by different tillage and weed management practices were recorded at definite intervals and the data tabulated in table 4 .

In rice-chickpea cropping sequence, it is revealed from the result that the BSR rate continuously increased from sowing of the crop to 50 DAS and there after narrowed down a little upto harvest. The respiration rate was found maximum in conventional tillage and minimum in zero tillage in all the growth stages of crop. Conventional tillage was found significantly superior over minimum and zero tillage at 10 DAS. However, it was found only significant over zero tillage at 30 DAS. From 50 DAS onwards non-significant variation was observed in BSR due to application of different tillage systems. This observation is a close proximity with Singh $e t$ al., (2007), who found at lower depth soil $(7.5-15 \mathrm{~cm})$ under conventional tillage were found to show significantly higher respiration rate at post germination stage of crop. Loudyi et al., (1999) also expressed similar views and mentioned that basal soil respiration is usually higher under conventional tillage than under zero tillage, resulting in higher specific respiration under conventional tillage than under zero tillage.

The data showed that there was an increase in BSR rate from 0 to 50 DAS followed by a decrease upto harvest stage in hand weeded and weedy check plots. Under rice-chickpea cropping sequence application of imazethapyr at different doses and pendimethalin@ 1000 $\mathrm{g} \mathrm{ha}^{-1}$ significantly reduced the BSR rate soon after their application. The toxic effect of these chemicals was traced upto 10 DAS and there after a gradual increment BSR rate was noticed, which seems that the applied herbicides of pre-emergence stage started to degrade before 30 DAS. Higher dose of imazethapyr significantly reduced the BSR values over its lowest dose. However, effect of pendimethalin was found in between @ 80 and $90 \mathrm{~g} \mathrm{ha}^{-1}$ dose of imazethapyr. The postemergence application of imazethapyr exhibited its effect soon after its application and highest reduction in BSR rate was noticed at 50 DAS followed by 30 DAS. With the increasing doses of imazethapyr the BSR values had shown a decreasing trend at 50 DAS the post-emergence application of imazethapyr was found effective to reduce the BSR values in comparison to its application of pre-emergence stage. Sebiomo et al., (2010) who found that the herbicide treatments had significant effect on percent organic matter of the soils treated with herbicides, which reduced significantly as compared to control. The basal soil respiration in rhizosphere soil of chickpea found significantly lower in herbicide treated plots compared to hand weeded and weedy check plots in all the growth period of crop.

\section{Biochemical study (dehydrogenase enzyme activity)}

The result on Dehydrogenase activity (DA) as affected by different tillage and weed management practices were recorded at definite interval and tabulated in table 5 .

The data showed that in rice-chickpea cropping sequence, there was an increase in dehydrogenase enzyme activity (DHA) in chickpea rhizosphere soil from 0 to 50 DAS followed by a decrease upto harvest was noticed in all the tillage practices. Effect of different tillage systems significantly varied upto $30 \mathrm{DAS}$ and there after all the tillage practices found statistically equal. Highest DHA was observed in conventional tillage and lowest in zero tillage. Conventional tillage was found significantly superior over 
minimum and zero tillage system at 10 DAS. However, it was only significant over zero tillage at 30 DAS. The above study indicated that effect of tillage existed upto 30 DAS and after that due to settlement of soil particles, all the tillage system found equal. This observation is a close agreement with Chowdhury et al., (2014), who concluded that in conventional tillage system. Ferreira et al., (2000) also reported relatively higher availability of soil organic matter at lower soil organic matter at lower soil profile under conventional tillage which may be due to even distribution of crop residues and other nutrients throughout the plough zone.

In soil enzymatic study it was found that the dehydrogenase activity was found significantly higher in conventional tillage treatments compared to other tillage treatments. The activity was found lowest under zero tillage treatment. Mijangos et al., (2005) also concluded that biological parameters have great value as early and sensitive indicator of change in soil properties induced by different soil management strategies.

The data related to DHA in rice-chickpea cropping sequence revealed that the preemergence application of pendimethalin and imazethapyr significantly reduced DHA soon after their application and their effect on DHA was visualized upto 10 DAS. From 30 DAS onwards the DHA was shown an increasing trend, which may be due to the degradation of applied herbicide after 10 DAS. When imazethapyr was applied at post-emergence stage its toxic effect was noticed upto 50 DAS after its application i.e. 20 DAS. It was also observed that the DHA reduced with the increasing doses of imazethapyr, when applied at pre and post emergence stage. Application of pendimethalin@1000 $\mathrm{g} \mathrm{ha}^{-1}$ had shown a value of DHA, which was intermediate between @ 80 and $90 \mathrm{~g} \mathrm{ha}^{-1}$, imazethapyr at pre-emergence level. One hand weeding practice was found significantly superior over herbicide applied plots in all the growing stages of crop except harvest stage.

Shukla and Mishra (1997) also reported similar type of result who found dehydrogense activity tended to decrease on pre and post emergence application, but the end of the experimentation it recovered and even increased many folds. Sebiomo et al., (2010) who found that the herbicide treatments had significant effect on percent organic matter of the soils treated with herbicides, which reduced significantly as compared to control. The dehydrogenase enzyme activity in rhizosphere soil of chickpea found significantly lower in herbicide treated plots compared to hand weeded and weedy check plots in all the growth period of crop.

This might be due to available substrate and aerobic environment in soil ecosystem. Tripathi et al., (1993) also reported the similar results.

\section{References}

Ahlawat, A. and Saraf C.S., (1985). IT pays to control weeds in pulses, Weed abstract, 4: 11-13.

Anderson T.H and Domsch K.H. (1980). Quantities of plant nutrients in the microbial biomass of selected soils. Soil Sci, 1130:211-216.

Andrson, C.; Streibig J.C. and Hass, H. (1982). Soil properties affecting the distribution of 37 weed species in Danish fields. Weed Res, 31: 181-187.

Augustin, B. (2003). Urban areas - sources of pesticide contamination of surface water? P-166-169. In: Balder H, Strauch KH, Backhaus GF(Eds) Second International symposium on Plant Health in Urban Horticulture, Berlin, Germany. 
Bagment, L. (2000). Dynamic of the segetal element of weed flora in the Lower Volga region, Z Pflanzenk. Pflanzen 17: 85-90.

Bhalla, C.S.; Kurchania, S.P. and Paradkar, N.R. (1998). Herbicidal weed control in chickpea (Cicer arietinum L.). World Weeds, 5(1-2): 121-124.

Brady Nyle C. (1985). Aeration and biological activities. In: The nature and properties of soils. Eurasia Publishing House Ltd, New Delhi. pp. 260-261.

Chowdhury, T.; Bhambri, M.C. and Gupta, S.B. (2014). Changes in the properties of a rice field soil as influenced by tillage and weed management. Journal of Agriculture Issues, 41(1): 25-27.

Davis A.S.; Dixon P.M. and Liebman M. (2003). Using Matrix Models to determine cropping system effects on annual weed demography, Ecol. Appl, $14: 655-668$

FAI, (2003). Fertilizer Statistics, (2002-2003). The Fertilizer Association of India. pp. III. 13 and 51.

Ferreira, C.; Andrade, D.S.; Chueire, L.M.; Takemura, S.M. and Liungria, M. (2000). Tillage method and crop rotation effects on the population sizes and diversity of bradyrhizobia noduling soybean. Soil Biol. and Biochem, 32: 627-637.

Jackson, M.L. (1967). Soil Chemical Analysis. Prentice Hall of India, Pvt. Ltd. New Delhi. pp. 42-48.

Janusauskaite, D.; Kadziene, G. and Auskalniene, O. (2013). The effect of tillage system on soil microbiota in relation to soil structure. Pol. J. Environ. Stud, 22 (5): 1387-1391.

Knights, E. (1991). Chickpea. In New crops agronomy and Potential of alternative crop species, eds. Jessop, R.S. and Wright, R.L., PP. 27-38 (Inkata Press, Melbourne).

Loudyi, M.C. (1988). La Flora Adventice Des Legumineuses Cultivees Dans Le Plateeau De Meknes. In Proceedings of the Seminar on Food Legumes in Morocco. Settat, April 7-9, 1987.
Malik, R.K and Balyan, R.S. (1988). Effect of herbicides and planting dates in chickpea. Indian Journal of Weed Sci, 20 (2): 7581

Marshal, E.J. P.; Brown, V.K.; Boatman, N.D.; Lutman, P.J. W.; Squire, G.R. and Ward, L.K. (2003). The role of weeds in supporting biological diversity within crop field. Weed Res, 43:77-89.

Mijangos, I.; Perez, R.; Albizu, I. and Garbisu, C. 2005. Effects of fertilization and tillage on soil biological parameters. Enz. And Microbial Tech, 40(1): 100-106.

Rajput, A.S.; Choubey, N.K.; Shrivastava, G.K. and Tripathi, R.S. (1998). Nodulation, root growth and nitrogen uptake in late sown chickpea (Cicer arietinum L.) under varying irrigation schedule and Weed management Practices. World Weeds, $5: 233-237$

Sebiomo, A.; Ogundero, V.W. and Bankole, S.A. (2010). Effect of four herbicides on microbial population soil organic matter and dehydrogenase activity. Department of Biological Sciences, Tai Solarin University of Education, Ijagun Ijebuode, Ogun State, Nigeria.

Shukla, A.K. (1997). Effect of herbicides butachlor, fluchloralin, 2, 4-D and oxyfluorfen on microbial population and enzyme activities of rice field soil. Indian J. Ecol, 24: 189-192.

Singh, M.; Chandurkar, P.S. and Kumar, A. (2007). Weed management in rice based zero tilled sown wheat. Pakistan Journal of Weed Science Research, 13 (3\&4): 183-189.

Singh, D. and K.C. Sharma. (1990). Economics of soybean cultivation under different methods of weed control. Indian J. Weed Sci, 22 (1-2): 7-11.

Subba Rao, N.S. (1988). Soil Microbiology. Oxford and IBH Publishing Co., New Delhi.

Tripathi, B.; Kumar, C. P. C.; Rengarajan, T.; Chandurkar, P.S. and Vijayalakshmi. (1993). Pendimethalin, metolachlor, residues in soil and their effect on soil microflora, weed spectrum and green peas 
(Pisum sativum L.) yield. In: Integrated weed management for sustainable agriculture proceeding of an Indian Society of Weed Science International Symposium, Hisar, India, 18-20 (Vol. II): 98-100.

Vengris, J.; Drake, M.; Colby, W.B. and Bart, J. (1953). Chemical composition of weeds and accompanying crop plant. Agronomy Journal, 45: 213-218.

Gomez, K. A. and Gomez, A. A. (1984). Statistical procedures for agricultural research. J. Wiley and Sons, Singapore.

Goud, V.V.; Murade, N.B.; Khakre, M.S. and Patil, A.N. (2013). Efficacy of imazethapyr and quizalofop ethyl herbicides on growth and yield of chickpea. The Bioscan, 8 (3):1015-1018.

Verma, S.K.; Singh, S.B.; Meena, R.S.; Prasad, S.K.; Meena, R.S. and G. (2015). A review of weed management in india: The need of new directions for sustainable agriculture. The Bioscan, 10 (1):253-263.

Kewat, M. L. (2014). Improved weed management in Rabi crops. National Training on Advances in Weed Management, pp. 22-25.

Zimdahl, R. L. (2007). Fundamental of weed science. Academic Press, Burlington, MA.

Robert, E.; Blackshaw; Molnar, L. J. and Janzen, H. H. (2004). Nitrogen fertilizer timing and application method affect weed growth and competition with spring wheat. Weed Science, 52(4): 614-622.
Babu, M. B. B. and Jain, V. (2012). Effects of nitrogen on competition between wheat and grassy weeds. Indian J. Weed Science, 44: 53-57.

Dubey, R. P. (2014). Integrated weed management- an approach. In Training Manual Advance Training in Weed Management, held at DWSR, Jabalpur, India on 14-23 January, and pp. 19-21.

Pandey, I. B.; Dwivedi, D. K. and Pandey, R. K. (2006). Efficacy of herbicides and fertilizer management on weed dynamics of wheat. National Symposium on Conservation Agriculture and Environment, October 26-28, at BHU, Varanasi, India, pp. 336-337.

Lodha, A. K.; Thakur, R.; Tabasshum, S. and Singh, S. (2010). Effect of fertilizer and manure on weed incidence, depletion of nutrients by weeds and yield of soybean. Nat. Symp. On IWM in the Era of Climate Change, held at NAAS, New Delhi on 21-22 August. p. 67.

Das, T. K. and Yaduraju, N. T. (2008). Effect of soil solarization and crop husbandry practices on weed species competition and dynamics in soybean- wheat cropping system. Indian J. Weed Science, 40(1\&2): $1-5$.

Verma, S. K. (2014). Enhancing sustainability in wheat production though irrigation regimes and weed management practices in eastern Uttar Pradesh. The Ecoscane, 6: 115-119.

\section{How to cite this article:}

Anjum Ahmad, Tapas Chowdhury, Sudhir Kumar Taunk, Devendra Dewangan, and Singh, A.P. 2017. Pre and Post Emergence Application of Imazthapyr on N Uptake, Nodulation and Microbial Population of Chickpea Sown after Rice in Vertisols of C. G. Int.J.Curr.Microbiol.App.Sci. 6(6): 2844-2857. doi: https://doi.org/10.20546/ijcmas.2017.606.338 